\title{
Erratum: Lepton-mediated electroweak baryogenesis, gravitational waves and the $4 \tau$ final state at the collider
}

\author{
Ke-Pan Xie \\ Center for Theoretical Physics, Department of Physics and Astronomy, Seoul National University, \\ Seoul 08826, Korea \\ E-mail: kpxie@snu.ac.kr
}

ERRATUM TO: JHEP02(2021)090

ArXiv EPrint: 2011.04821 
Eq. (B.4) should be [1]

$$
\begin{aligned}
\Gamma_{Y}= & \frac{3 y_{\tau}^{2}}{4 \pi^{3} T_{n}^{2}}\left(m_{h}^{2}-m_{\ell}^{2}-m_{\tau}^{2}\right) \int_{m_{\tau}}^{\infty} d \omega_{R} h_{F}\left(\omega_{R}\right) \ln \left(\frac{e^{\omega_{R} / T_{n}}+e^{\omega_{-} / T_{n}}}{e^{\omega_{R} / T_{n}}+e^{\omega_{+} / T_{n}}} \frac{e^{\omega_{+} / T_{n}}-1}{e^{\omega_{-} / T_{n}}-1}\right) \\
& +\frac{3 \zeta_{3}}{32 \pi^{3}} g^{2} y_{\tau}^{2} T_{n}\left[\left(1+\frac{1}{6} \tan ^{2} \theta_{W}\right) \ln \frac{8 T_{n}^{2}}{m_{\ell}^{2}}+\frac{2}{3} \tan ^{2} \theta_{W} \ln \frac{8 T_{n}^{2}}{m_{\tau}^{2}}\right],
\end{aligned}
$$

while in its original version the second line was mistaken to be $\propto g_{s}^{2}$. After correcting this, the washout effect from Yukawa interaction is weakened and hence the generated $\eta_{B}$ in the right panel of figure 2 increases by $1 \% \sim 20 \%$, depending on $v_{w}$. The improved figure 2 (right panel) is as follows.

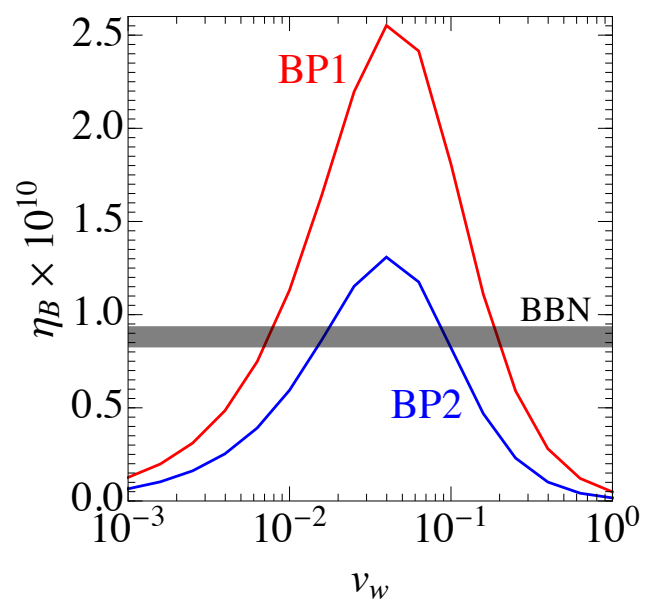

I thank Benoit Laurent for pointing out this to me, and Yehonatan Viernik for the useful discussions.

Open Access. This article is distributed under the terms of the Creative Commons Attribution License (CC-BY 4.0), which permits any use, distribution and reproduction in any medium, provided the original author(s) and source are credited.

\section{References}

[1] M. Joyce, T. Prokopec and N. Turok, Nonlocal electroweak baryogenesis. Part I. Thin wall regime, Phys. Rev. D 53 (1996) 2930 [hep-ph/9410281] [INSPIRE]. 